\title{
Elastic properties of model porous ceramics *
}

\author{
Anthony P. Roberts ${ }^{\ddagger} \dagger$ and Edward J. Garboczi ${ }^{\ddagger}$ \\ ${ }^{\ddagger}$ Building Materials Division, \\ National Institute of Standards and Technology, Gaithersburg, MD 20899, USA \\ ${ }^{\dagger}$ Centre for Microscopy and Microanalysis, \\ University of Queensland, St. Lucia, Queensland 4072, Australia
}

June 20, 2000

\begin{abstract}
The finite element method (FEM) is used to study the influence of porosity and pore shape on the elastic properties of model porous ceramics. The Young's modulus of each model was found to be practically independent of the solid Poisson's ratio. At a sufficiently high porosity, the Poisson's ratio of the porous models converged to a fixed value independent of the solid Poisson's ratio. The Young's modulus of the models is in good agreement with experimental data. We provide simple formulae which can be used to predict the elastic properties of ceramics, and allow the accurate interpretation of empirical property-porosity relations in terms of pore shape and structure.
\end{abstract}

\section{Introduction}

The elastic properties of two-phase (solid-pore) porous materials depend on the geometrical nature of the pore space and solid phase, as well as the value of porosity [1, 2, 3, 4]. Relevant aspects of porous materials may include pore shape and size as well as the size and type of the interconnections between solid regions. These features, which generally lack precise definition, comprise the microstructure of the material. In order to predict properties, or properly interpret experimental property-porosity relationships, it is necessary to have an accurate method of relating elastic properties to porosity and microstructure. In this paper we use the finite element method to derive simple formulae that relate Young's modulus and Poisson's ratio to porosity and microstructure, for three different models of microstructure.

There have been several different approaches to deriving property-porosity relations for porous materials. Formulae derived using the micro-mechanics method [5, 6, 7] are essentially various methods of approximately extending exact results for small fractions of spherical or ellipsoidal pores to higher porosities. This includes the differential [8] and self consistent methods [9, 10, 11, 12] as well as the commonly used semi-empirical correction to the dilute result made by Coble and Kingery [1]] to explain the properties of porous alumina. A drawback of this approach is that the microstructure corresponding to a particular formula is not precisely known; hence agreement or disagreement with data can neither confirm nor reject a particular model. A second problem is that these types of models provide no predictions for the case where the microstructure is comprised of incompletely sintered grains, which is a common morphology in porous ceramics. A second class of results [3, 13] have been termed minimum solid area (MSA) models. In this approach purely geometrical reasoning is used to predict the elastic moduli based on the weakest points within the structure. Again, the microstructure that corresponds to the MSA predictions is not exactly known. A number of semi-empirical relations have also been proposed [2], which generally provide a reasonable means of describing data, extrapolating

* Submitted to the Journal of the American Ceramic Society 
results and comparing data among materials. However, lacking a rigorous connection with microstructure, these results do not offer either predictive or interpretive power. Theoretical bounds [5], 14] exist for the elastic properties, but the vanishing of the lower bound for porous materials lessens their predictive power when the upper bound does not provide a good estimate. There are numerous other approaches, including the generalized method of cells [6, 他.

Another approach is to computationally solve the equations of elasticity for digital models of microstructure [15, 16]. In principle this can be done exactly. However, large statistical variations and insufficient resolution, have limited the accuracy of results obtained to date. Only recently have computers been able to handle the large three-dimensional models and number of computations needed to obtain reasonable results. As input to the method, we employ three different microstructural models that broadly cover the types of morphology observed in porous ceramics. The models are based on randomly placed spherical pores, solid spheres, and ellipsoidal pores [14]. The centers of the pores or solid particles are un-correlated which leads to realistic microstructures in which both the pore and solid phase are interconnected. The results, which can be expressed simply by two (or sometimes three) parameter relations, correspond to a particular microstructure and explicitly show how the properties depend on the nature of the porosity. Therefore, the results can be used as a predictive tool for cases where the microstructure of the ceramic is similar to one of the models, and as an interpretive tool if the microstructure is unknown. The numerically exact FEM results are compared with various well-known micro-mechanics and MSA results to determine how close an approximation a particular formula provides for each model. In the FEM, we can freely vary the properties of the solid phase, allowing us to determine the dependence of Young's modulus and Poisson's ratio on the solid Poisson's ratio as well as on the porosity. This question has attracted recent interest in the ceramics literature [17, 18, 19].

\section{Computational Results}

A microstructure made up of a digital image is already naturally discretized and so lends itself to numerical computation of many quantities. The finite element method uses a variational formulation of the linear elastic equations, and finds the solution by minimizing the elastic energy via a fast conjugate gradient method. The digital image is assumed to have periodic boundary conditions. Details of the theory and programs used are reported in the papers of Garboczi \& Day [15] and Garboczi [20].

In order to obtain accurate results using the FEM on models of random porous materials, it is absolutely necessary to estimate and minimize three sources of error: finite size effects, discretization errors, and statistical fluctuations. This has generally not been done in the past, owing to limitations in computer memory and speed. FEM results for random microstructures do not have much meaning without such an error analysis.

The various sources of error are defined in the following way. First, the length scale of the microstructure is fixed, usually by fixing the size of a typical pore (e.g. the spherical pore radius). The size of the system is then controlled by the side length of the cubic sample, denoted $T$. The size of $T$ compared to the pore size controls how many pores will appear in the computational cell. A real material has many thousands or more such pores. Errors can occur in using a smaller number in a periodic cell to simulate a much larger number. We vary $T$ in order to map out this effect.

Once a value of $T$ is chosen that minimizes finite size errors but is still computationally possible, we next must consider the discretization error, which comes about because we are using discrete pixels to represent continuum objects. The number of pixels along each edge of the cubical unit cell is $M$, giving a resolution of $d x=T / M$ (in units of $\mu \mathrm{m}$ per pixel, if $T$ is in $\mu \mathrm{m}$ ). For the chosen value of $T$, a value of $M$ is chosen that also gives acceptable discretization errors, usually on the order of a few percent.

Finally, when computing the properties of random materials, either computationally or experimentally, one must carefully choose the number of samples $\left(N_{s}\right)$ over which the results need to be averaged 
to produce acceptable uncertainties. This value is again chosen, within computational constraints, to keep statistical fluctuations within a few percent.

Overlapping Solid Spheres. Realizations of the overlapping solid sphere model [14, 21] are generated by placing solid spheres at random points in the unit cell. This produces a set of overlapping grains that mimic the microstructure of sintered ceramic composites (see Fig. 17a). The space outside the solid grains is the pore space, with porosity $\phi$. The pore phase is macroscopically connected above porosities of $\phi \approx 0.03$ and the solid phase remains connected for values of $\phi$ below $\approx 0.70$ [14]. Above $\phi=0.7$, the solid phase is composed of isolated solid particles. So between $\phi=0.03$ and $\phi=0.70$, the overlapping solid sphere model is bi-continuous. In ceramics the porosity is generally less then 0.40 , in this bi-continuous regime. We therefore consider the elastic properties for $0.1 \leq \phi \leq 0.50$, where the solid Poisson's ratio, $\nu_{s}$, varied over the range $0.1 \leq \nu_{s} \leq 0.4$.

To generate the microstructure we chose solid spheres of radius $r=1 \mu \mathrm{m}$. Note that the elastic properties are length scale invariant so the results apply to spheres of any radius for which the continuum assumption holds. A preliminary study showed that finite size errors were acceptably small for cubic samples with edge length $T=12 \mu \mathrm{m}$. To study the discretization errors we generated one realization of the model with porosity $\phi=0.5$ at $M=48 \ldots 128$. The elastic properties depend quite strongly on resolution. We found that the variation of Young's modulus with $M$ could be described by the relation [22]

$$
E_{\mathrm{FEM}}(M) \approx E_{0}+a M^{-1}
$$

where $E_{0}$ can be identified as the continuum value (corresponding to infinitely large $M$ ). The same is true for Poisson's ratio. Even at $M=128$ the finite element code overestimates the 'exact' result for the Young's modulus by $30 \%$. Therefore, for the overlapping sphere model it is necessary to measure the elastic moduli at three different values of $M$ and extrapolate the results to $M \rightarrow \infty$. We chose $N_{s}=5$ samples at each resolution and porosity, except at $\phi=0.5$ where large statistical variations implied a larger number of samples was necessary $\left(N_{s}=10\right)$. Thus 30 different realizations of the models were considered, each at 3 different discretizations, for a total of 90 models.

The statistical variation in Young's modulus and Poisson's ratio for the case $\nu_{s}=0.2$ are shown in Table 1. The error bars shown in the table are equal to twice the standard error (S.E. $=\sigma / \sqrt{N_{s}}$ with $\sigma$ the standard deviation). Therefore there is a 95\% chance that the "true" result lies between the indicated error bars. The results are accurate to within $20 \%$ at $\phi=0.5$; the error decreasing with porosity to less than $10 \%$ for $\phi \leq 0.30$. The expected Gaussian distribution of the measured averages implies that the results are actually more accurate than this. For example, the anticipated relative errors are halved if a $68 \%$ likelihood threshold is used (i.e., \pm one standard error).

In addition to the above results we also computed the elastic moduli of the 90 model microstructures at solid Poisson's ratios $\nu_{s}=0.1,0.3$ and 0.4 . The statistical variation was not significantly different from the case $\nu_{s}=0.2$. Combined with the data for $\nu_{s}=0.2$ this covers most commonly occuring
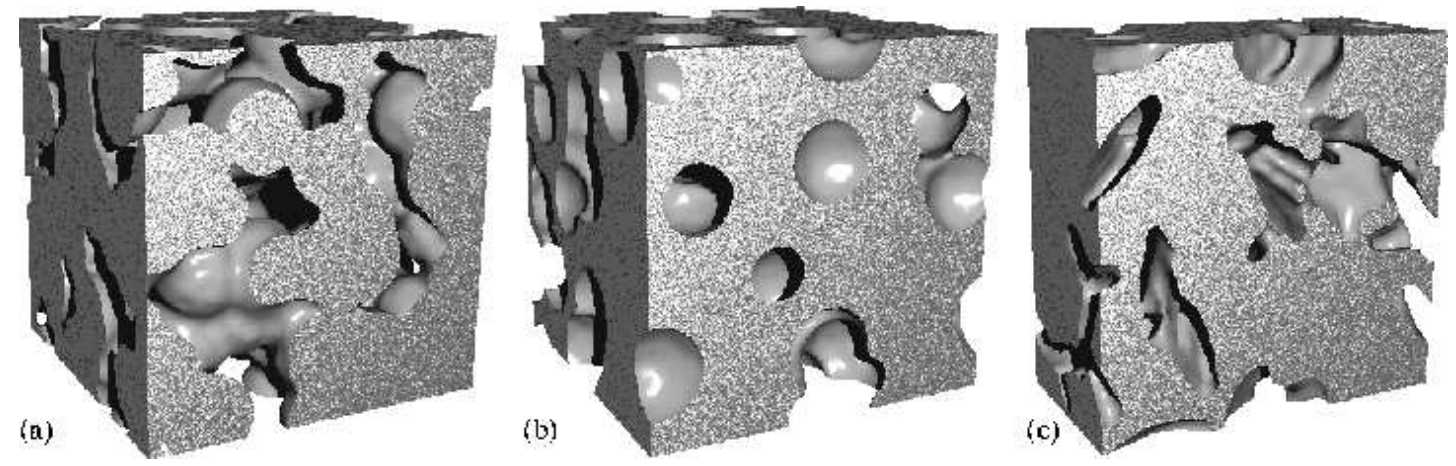

Figure 1: Showing pieces of the various models studied: (a) overlapping solid spheres, (b) overlapping spherical pores, and (c) overlapping ellipsoidal pores. 
Table 1: Elastic properties of the three models $\left(\nu_{s}=0.2\right)$.

\begin{tabular}{|c|c|c|c|c|c|c|}
\hline & \multicolumn{2}{|c|}{ Overlapping solid spheres } & \multicolumn{2}{c|}{ Overlapping spherical pores } & \multicolumn{2}{c|}{ Overlapping ellipsoidal pores } \\
\hline$\phi$ & $E / E_{s}$ & $\nu$ & $E / E_{s}$ & $\nu$ & $E / E_{s}$ & $\nu$ \\
\hline 0.1 & $0.71 \pm 1 \%$ & $0.19 \pm 1 \%$ & $0.80 \pm 1 \%$ & $0.20 \pm 1 \%$ & $0.73 \pm 2 \%$ & $0.19 \pm 3 \%$ \\
0.2 & $0.47 \pm 2 \%$ & $0.18 \pm 4 \%$ & $0.62 \pm 2 \%$ & $0.20 \pm 2 \%$ & $0.52 \pm 3 \%$ & $0.18 \pm 4 \%$ \\
0.3 & $0.25 \pm 6 \%$ & $0.17 \pm 9 \%$ & $0.46 \pm 3 \%$ & $0.21 \pm 3 \%$ & $0.34 \pm 4 \%$ & $0.18 \pm 6 \%$ \\
0.4 & $0.12 \pm 13 \%$ & $0.15 \pm 25 \%$ & $0.33 \pm 4 \%$ & $0.21 \pm 4 \%$ & $0.20 \pm 3 \%$ & $0.18 \pm 4 \%$ \\
0.5 & $0.039 \pm 22 \%$ & $0.15 \pm 21 \%$ & $0.21 \pm 8 \%$ & $0.22 \pm 9 \%$ & $0.11 \pm 4 \%$ & $0.18 \pm 6 \%$ \\
\hline
\end{tabular}

solids. The scaled Young's modulus for each value of $\nu_{s}$ is plotted against porosity in Fig. Q. Remarkably, the scaled Young's modulus of the porous material appears to be practically independent of $\nu_{s}$. This result has been proven to be exact in 2-D [23, 24] and appears to hold to a very good approximation in 3-D. We found that the Young's modulus data are well described by an equation of the form

$$
\frac{E}{E_{s}}=\left(1-\frac{\phi}{\phi_{0}}\right)^{n}
$$

with $n=2.23$ and $\phi_{0}=0.652$ and $0 \leq \phi \leq 0.5$. Note that $n$ and $\phi_{0}$ are empirical correlation parameters and should not be interpreted as the percolation exponent and threshold, respectively. Percolation concepts are generally valid closer to the threshold $\phi_{c} \approx 0.7$ (for this model) and a higher value of $n$ is expected. The computational cost of accurately measuring the elastic properties increases greatly as the percolation threshold is approached.

The Poisson's ratio of the porous material is shown in Fig. 3 as a function of $\phi$ and $\nu_{s}$, and appears to be a flow diagram [23], where the Poisson's ratio asymptotically approaches a fixed point, independently of the value of the solid Poisson's ratio. This flow diagram has been analytically proven to hold in 2-D, when a percolation threshold exists at which the Young's modulus goes to zero [23, 24]. This flow diagram also appears to be valid in 3-D as well, within numerical uncertainty. The Poisson's ratio data shown in Fig. 3 can be roughly described by the simple linear relation,

$$
\nu=\nu_{s}+\frac{\phi}{\phi_{0}}\left(\nu_{0}-\nu_{s}\right)=\nu_{0}+\left(1-\frac{\phi}{\phi_{0}}\right)\left(\nu_{s}-\nu_{0}\right)
$$

with two fitting parameters $\nu_{0}=0.140$ and $\phi_{0}=0.472$. A more accurate fit is obtained with the three parameter relation,

$$
\nu=\nu_{0}+\left(1-\frac{\phi}{\phi_{0}}\right)^{m}\left(\nu_{s}-\nu_{0}\right)
$$

with $\nu_{0}=0.140, \phi_{0}=0.500$ and $m=1.22$.

Overlapping Spherical Pores. The overlapping spherical pore (or swiss cheese) model [14, 21] is generated by interchanging the roles of the solid and pore phase of the overlapping solid sphere model (see Fig. 1 1 b). The morphology corresponds to isolated spherical pores at low porosity, with the pores becoming macroscopically interconnected at $\phi \approx 0.3$. The solid phase remains connected up to $\phi \approx$ 0.97. This type of morphology may arise in ceramics generated with a particulate filler [1], or where bubbles form in a molten state [25]. We consider solid Poisson's ratios in the range $-0.1 \leq \nu_{s} \leq 0.4$.

We determined that statistical errors were acceptable for a computational cube of size $T=12 \mu \mathrm{m}$ with pores of radii $r=1 \mu \mathrm{m}$. Using $M=80$ pixels, the discretization errors were less than $3 \%$ for $\phi=0.5$ and $2 \%$ for $\phi=0.3$. Therefore, it was not considered necessary to generate samples at different discretizations $(M)$ and extrapolate the results. As for solid spheres, the Young's modulus was independent of the solid Poisson's ratio to a very good approximation. The Young's modulus can be described by Eq. (2) with $n=1.65$ and $\phi_{0}=0.818$ (Fig. (2). Poisson's ratio of the porous material 


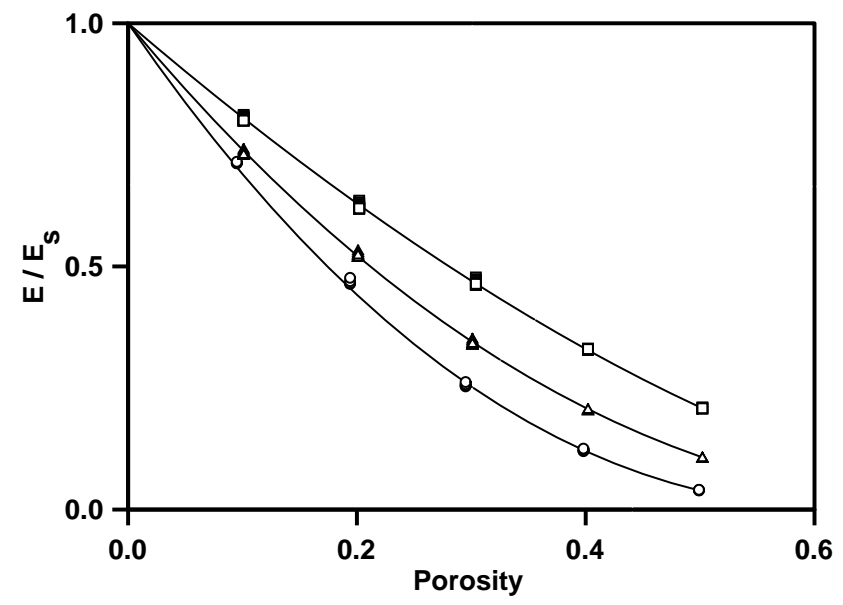

Figure 2: The Young's modulus of the three microstructure models. The solid lines are empirical fits to the equation $E / E_{s}=\left(1-\phi / \phi_{0}\right)^{n}$. Data is shown for overlapping solid spheres $\left(\circ, n=2.23, \phi_{0}=\right.$ $0.652)$, spherical pores $\left(\square, n=1.65, \phi_{0}=0.818\right)$ and ellipsoidal pores $\left(\triangle, n=2.25, \phi_{0}=0.798\right)$ for $\nu_{s}=-0.1, \ldots, 0.4$. Note that the $E$ is practically independent of the solid Poisson's ratio in each case [the different values of $E\left(\nu_{s}\right)$ at each porosity are almost indistinguishable].

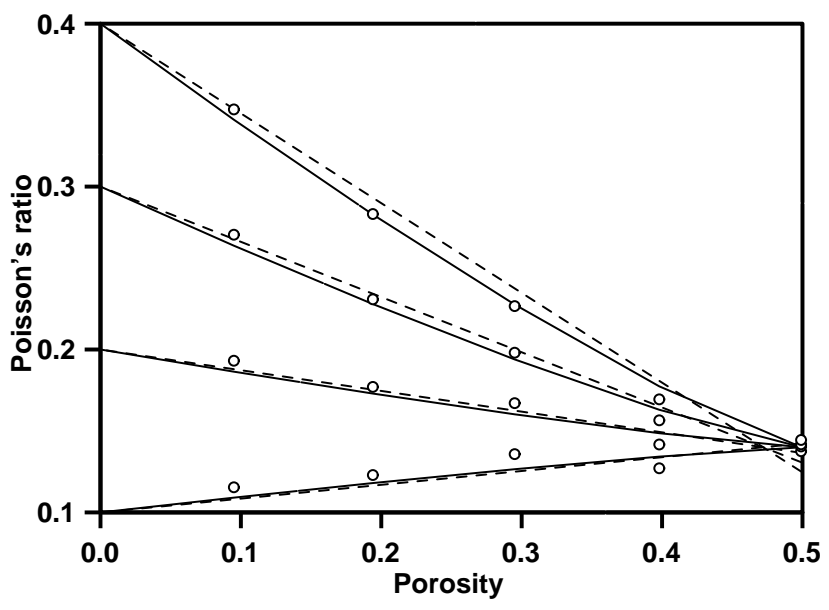

Figure 3: The Poisson's ratio of the overlapping solid sphere model as a function of porosity for $\nu_{s}=0.1-0.4$. The dashed lines are an empirical fit to Eq. (3). The solid lines correspond to the three parameter relation given in Eq. ( 4 ), with the value of all parameters given in the text. The intercepts of the lines at zero porosity correspond to the solid Poisson's ratio.

is shown in Fig. 7 and is simply described by the linear relation given in Eq. (3) with $\nu_{0}=0.221$ and $\phi_{0}=0.840$. Again, a flow diagram is observed.

Overlapping Ellipsoidal Pores. A common method of analyzing the effect of pore shape on elastic properties is to study ellipsoidal pores. In analytic formulae, it is possible to treat the limiting cases of needles and platelets, although the difficulty of resolving these fine structures prohibits these limits from being treated with the finite element method. However, the percolation properties of these limiting cases can be computationally studied [26]. To gauge the effect of deviations from spherical shaped pores we considered isotropically oriented overlapping oblate ellipsoidal pores bounded by the surface $(x / a)^{2}+(y / b)^{2}+(z / c)^{2}=1$ with $a=b=1 \mu \mathrm{m}$ and $c=0.25 \mu \mathrm{m}$ (see Fig. 11c). For this case, the pore phase becomes connected at porosity $\phi=0.2$ [26]. Statistical errors were found to be 


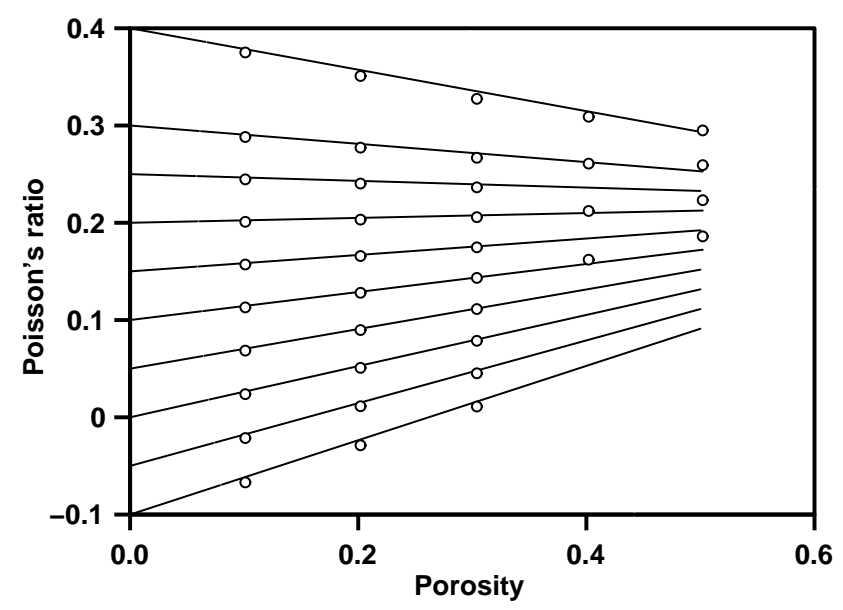

Figure 4: Poisson's ratio of the overlapping spherical pore model as a function of solid Poisson's ratio and porosity. The lines are an empirical fit to the relation $\nu=\nu_{s}+\phi / \phi_{0} \times\left(\nu_{0}-\nu_{s}\right)$ with $\nu_{0}=0.221$ and $\phi_{0}=0.840$. The intercepts of the lines at zero porosity correspond to the solid Poisson's ratio.

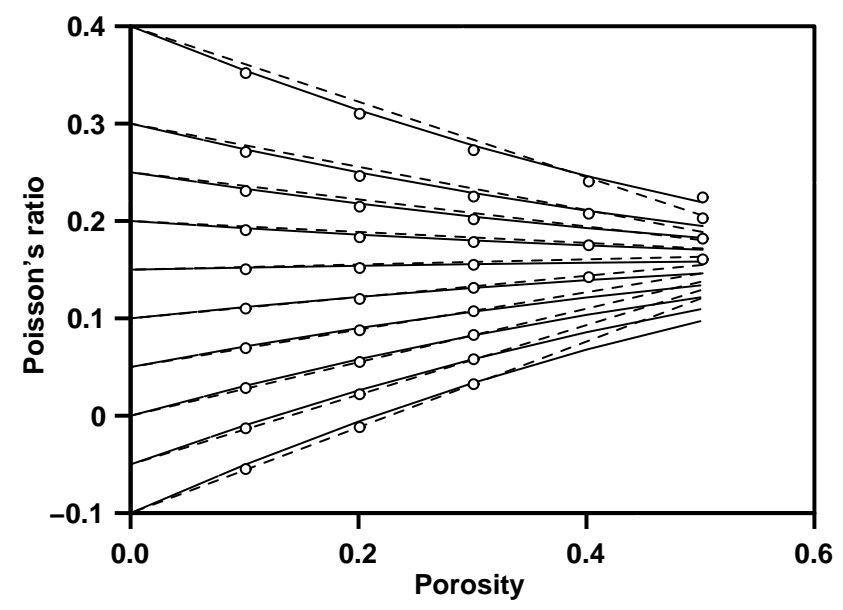

Figure 5: Poisson's ratio of the overlapping ellipsoidal pore model as a function of solid Poisson's ratio and porosity. The solid lines are an empirical fit to Eq. (4) and the dashed lines correspond to the linear fit to Eq. (3) with $\nu_{0}=0.166$ and $\phi_{0}=0.604$. The intercepts of the lines at zero porosity correspond to the solid Poisson's ratio.

acceptable for a computational cube of size $T=10 \mu \mathrm{m}$. Using $M=96$ pixels the discretization errors were $3 \%$ for $\phi=0.5$ and $2 \%$ for $\phi=0.3$. As for the case of spherical pores, these errors were considered sufficiently small, so that the added computational burden of the extrapolation technique could be again avoided.

Again the Young's modulus was found to be independent of the solid Poisson's ratio to a very good approximation. The results, shown in Fig. 2, can be accurately described by Eq. (2) with $n=2.25$ and $\phi_{0}=0.798$. The Poisson's ratio (Fig. 5) can be roughly fit using the Eq. (3) with $\nu_{0}=0.166$ and $\phi_{0}=0.604$. A better fit is obtained using Eq. (4) with $m=1.91, \nu_{0}=0.161$ and $\phi_{0}=0.959$. A flow diagram similar to that seen before is obtained.

The CPU time and memory required for these computations are an important "experimental" detail. The memory requirement for a given model was $230 \times M^{3}$ bytes, where $M$ was the edge length in pixels of a cubic unit cell. So for the largest computations carried out, $M=128$, the memory 
requirement was about 500 Mbytes. The amount of CPU time consumed was approximately 3000 hours, divided among different modern workstations.

\section{Comparison with micro-mechanical and MSA formulae}

In this section we compare a selection of well known theoretical results with the 'numerically exact' data computed in the previous section. These results include both analytically exact results (bounds, expansions, dilute limits, composite sphere assemblage), as well as approximate results (effective medium theories, minimum solid area models).

There are several kinds of exact bounds that have been derived for elastic materials [5, 14]. These are equations involving the different phase moduli, the volume fractions of the different phases, and various correlation functions that define the geometry of the composite. The upper bound gives the maximum possible composite elastic moduli, and the lower bound gives the lowest possible composite elastic moduli. The bounds used in this paper are three- point bounds, which have been written out explicitly for overlapping solid spheres and overlapping spherical pores [14]. In the case where one phase has zero elastic moduli, as is true in this paper, the lower bound becomes zero as well, and so only the upper bound is meaningful.

An exact perturbation expansion also exists, where the elastic moduli of a two-phase material is expanded in terms of parameters involving the individual elastic moduli of each phase and geometrical quantities [27, 28]. This expansion has been carried out to three terms explicitly, and it is this truncated form to which we will compare our numerical data. The result is expected to be accurate when the void phase is not interconnected.

Another exact result, which is used later in this section to build the various effective medium theories, is the case of dilute spherical pores for which the exact effective moduli are given by

$$
\begin{aligned}
K & =K_{m}+c_{i} P^{m i}\left(K_{i}-K_{m}\right) \\
G & =G_{m}+c_{i} Q^{m i}\left(G_{i}-G_{m}\right)
\end{aligned}
$$

with

$$
\begin{aligned}
P^{m i} & =\frac{3 K_{m}+4 G_{m}}{3 K_{i}+4 G_{m}}, \quad Q^{m i}=\frac{G_{m}+F_{m}}{G_{i}+F_{m}} \\
F_{m} & =\frac{G_{m}}{6} \frac{9 K_{m}+8 G_{m}}{K_{m}+2 G_{m}} .
\end{aligned}
$$

Here $c_{i}$ denotes the concentration (volume fraction) of inclusions and the subscripts $i$ and $m$ on the bulk $K$ and shear modulus $G$ denote the properties of the inclusion and matrix, respectively. The result is attributed to numerous authors [5]. For a porous matrix $K_{i}=G_{i}=0$ and the porosity is $\phi=c_{i}$. The result is strictly valid for small concentrations of inclusions $\phi \ll 1$ (in practice $\phi<0.1$.) Cast in terms of the engineering constants for porous inclusions this result becomes

$$
\begin{aligned}
E & =E_{m}-\frac{3}{2} \phi E_{m} \frac{9-4 \nu_{m}-5 \nu_{m}^{2}}{7-5 \nu_{m}}+O\left(\phi^{2}\right) \\
\nu & =\nu_{m}-\frac{3}{2} \phi \frac{\left(5 \nu_{m}-1\right)\left(1-\nu_{m}^{2}\right)}{7-5 \nu_{m}}+O\left(\phi^{2}\right)
\end{aligned}
$$

Our prior statement[29] of Eq. (8) inadvertently omitted the factor of 3/2, although the correct result was used in the paper. A non zero quadratic term can be added (as an empirical correction) to ensure that $E=0$ at $\phi=1$. This was suggested by Coble and Kingery [1] for MacKenzie's [30] result for spherical pores, which is equivalent to Eqs. (5)-(7) with $K_{i}=G_{i}=0$.

To adapt the dilute formulas to the case of finite porosity a number of proposals have been made. The approximate equations that result are usually called effective medium theories. The most common approximation is the so-called self consistent method (SCM) of Hill [9] and Budiansky [10]. In 
this model the equations of elasticity are solved for a spherical inclusion embedded in a medium of unknown effective moduli. The effective moduli $K$ and $G$ are then derived. In the dilute case the embedding medium is just the matrix. The Hill-Budiansky result can be stated as [12]

$$
\begin{aligned}
c_{i} P^{* i}\left(K_{i}-K_{*}\right)+c_{m} P^{* m}\left(K_{m}-K_{*}\right) & =0 \\
c_{i} Q^{* i}\left(G_{i}-G_{*}\right)+c_{m} Q^{* m}\left(G_{m}-G_{*}\right) & =0
\end{aligned}
$$

where $K_{*}$ and $G_{*}$ denote the effective moduli and $P^{* m}$ and $Q^{* m}$ are given in Eq. (7). The equations cannot be explicitly solved and numerical methods are necessary (see Hill [9] and Berryman [12] for details). In the case of porous inclusions, the moduli vanish at $\phi=\frac{1}{2}$, which is a property not shared with most composites (e.g., the overlapping sphere model). To derive a more realistic result, Christensen and Lo [31] generalized the SCM (GSCM) to the case of a spherical shell embedded in a matrix of unknown moduli. The result is complicated and not reproduced here.

The differential method (reviewed by McLaughlin [8]) provides an alternative model using a similar philosophy. Suppose that the effective moduli of a composite medium are known to be $K_{*}$ and $G_{*}$. Now if a small additional concentration of inclusions are added, the change in $K_{*}$ and $G_{*}$ is approximated to be that which would arise if a dilute concentration of inclusions were added to a uniform, homogeneous matrix with moduli $K_{*}$ and $G_{*}$. This leads to a pair of coupled differential equations,

$$
\begin{aligned}
\frac{d K_{*}}{d c_{i}} & =P^{* i} \frac{K_{i}-K_{*}}{1-c_{i}} ; K_{*}\left(c_{i}=0\right)=K_{m} \\
\frac{d G_{*}}{d c_{i}} & =Q^{* i} \frac{G_{i}-G_{*}}{1-c_{i}} ; G_{*}\left(c_{i}=0\right)=G_{m} .
\end{aligned}
$$

The dilute result, the self consistent result [12], and the differential method [8] can all be extended to the case of spheroidal inclusions. The general results [11] for $P^{m i}$ and $Q^{m i}$ have been given by Berryman [12]. In addition to these results $\mathrm{Wu}$ [11] derived a variant of the self consistent method, where $K_{*}$ and $G_{*}$, the effective moduli, are found by implicitly solving the equations

$$
\begin{aligned}
K_{*} & =K_{m}+c_{i} P^{* i}\left(K_{i}-K_{m}\right) \\
G_{*} & =G_{m}+c_{i} Q^{* i}\left(G_{i}-G_{m}\right) .
\end{aligned}
$$

A different type of microstructure is provided by Hashin's [32, 5] model of space-filling polydisperse hollow spheres (the "composite-sphere assemblage"). Although a simple formula exists for the bulk modulus over the full porosity range [32], exact results for the Young's moduli are not available. Ramakrishnan and Arunachalam [33] recently derived the approximation

$$
\begin{aligned}
\frac{E}{E_{s}} & =\frac{(1-\phi)^{2}}{\left(1+2 \phi-3 \nu_{s} \phi\right)} \\
\nu & =\frac{\left(4 \nu_{s}+3 \phi-7 \nu_{s} \phi\right)}{4\left(1+2 \phi-3 \nu_{s} \phi\right)} .
\end{aligned}
$$

However, the derivation is not rigorous. In particular, the exact result for the bulk modulus of the model [32] is around twice that predicted by Eqs. (16-17) at $\phi=0.5$. Since Eq. (16) was found to provide reasonable agreement with experimental data for porous ceramics [17], we compare its predictions to our FEM data below.

The final class of results we consider is provided by the 'minimum solid area' (MSA) models [34] (which have been recently reviewed by Rice [3, 13]). This approach is based on the assumption that the ratio of the effective moduli to the solid moduli is directly proportional to the minimum ratio of solid contact area to the total cross-sectional area of periodic structures. The approximation derived depends on the particular model considered. We consider two basic models most closely aligned with our FEM data: simple cubic arrays of solid and porous spheres. The latter case provides a particularly 


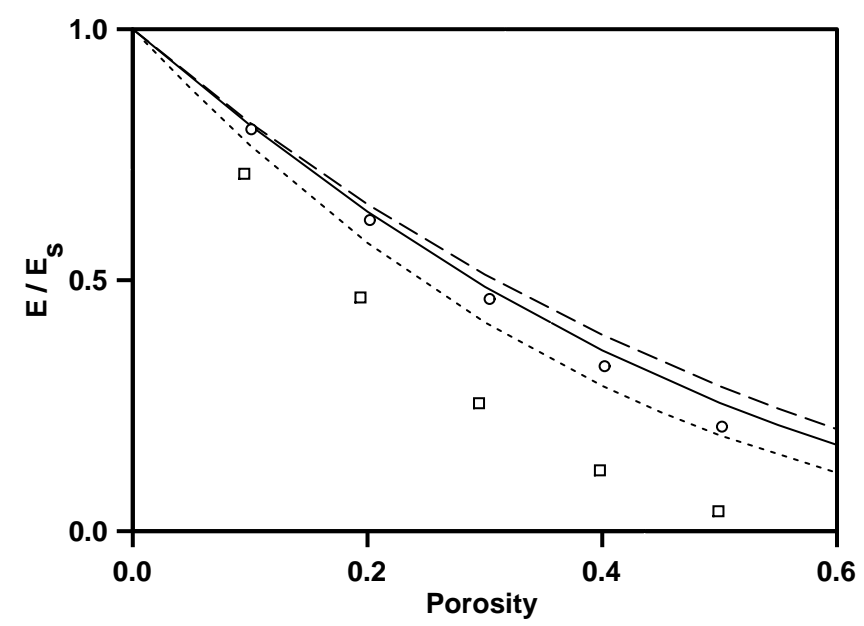

Figure 6: A comparison of rigorous bounds and expansions to the FEM data for overlapping spherical pores ( $(\circ)$ and overlapping solid spheres $(\square)$. The truncated expansion $(-)$ and the three-point upper bound (-- ) are shown for the spherical pore case. Only the three-point bound $(\cdots)$ is shown for the solid sphere case. The three-point lower bound is zero for porous materials. The Poisson's ratio is 0.2 for all the results.

simple example of the type of result which can be derived. Suppose the repeat distance of the lattice is $2 h$ and the sphere radius is $r$. The Young's modulus is assumed to be proportional to the area fraction, giving

$$
\frac{E}{E_{s}}=\frac{(2 h)^{2}-\pi r^{2}}{(2 h)^{2}}=1-\frac{\pi}{4}\left(\frac{6}{\pi}\right)^{\frac{2}{3}} \phi^{\frac{2}{3}}
$$

since $\phi=\frac{1}{6} \pi(r / h)^{3}$. The form of the result changes for $r>h$ (or $\phi>\pi / 6=0.52$ ) as the spheres begin to coalesce. Rice [3] has noted the results of many different periodic structures can be approximated by the form $E / E_{s}=e^{-b \phi}$ over a range of porosities. For example, $b \approx 5$ for the solid sphere model and $b \approx 3$ for the porous sphere model. It is argued that for a given set of data, $b$ can be compared with known values to assess the type of porosity. Often fractions of different types of porosity are assumed to match experimental data making the method an interpretive rather than a predictive tool. Since we have measured $E$ for microstructures based on solid sphere contacts and porous spheres we should be able to ascertain the accuracy of the MSA formulae for these cases.

Fig. 6 shows the comparison between the exact three-point bounds [14] for the overlapping solid sphere and spherical pore case, the truncated expansion [27, 28] for the overlapping spherical pore case, and the numerical results. Clearly the expansion does better than the three-point bound for the overlapping spherical pore case, though both are fairly close to the numerical results. The bound lies far away from the overlapping solid sphere numerical results, however. For this case, the truncated expansion does not exist. Only the $\nu_{s}=0.2$ data is shown. Using the truncated expansion, one can show that in 3-D, the Young's modulus is not exactly independent of the solid Poisson's ratio, but is rather a very good approximation, as was shown earlier in this paper.

In Fig. 7, we compare the FEM data $\left(\nu_{s}=0.2\right)$ for overlapping spherical pores with dilute and effective medium theory analytic results. At this Poisson's ratio the SCM and dilute results reduce to $E / E_{s}=1-2 \phi$ while the differential and dilute results with the Coble-Kingery correction reduce to $E / E_{s}=(1-\phi)^{2}$. Since the analytic results are based on the case of dilute spherical pores they all match the FEM data at $\phi=0.1$. The dilute and SCM results under-estimate the FEM data at higher porosities because of the aphysical percolation threshold at $\phi=\frac{1}{2}$. The generalized SCM overestimates the data, while the differential method performs reasonably well over the entire porosity 


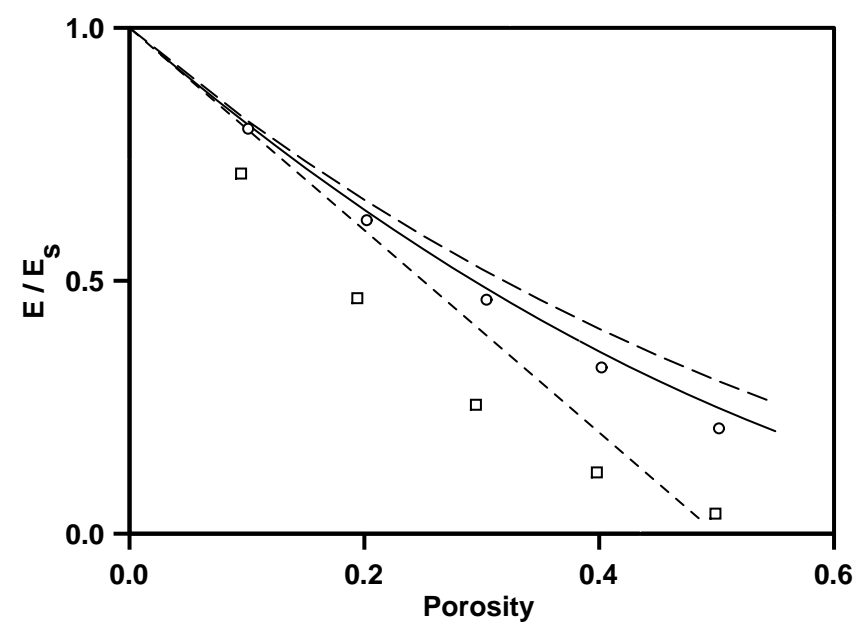

Figure 7: A comparison of different theories to the FEM data for overlapping spherical pores (o). The lines correspond to the dilute result and self consistent method [9, 10] (or SCM) (- - ), the differential method [8] and dilute result with Coble-Kingery correction ( - ) and the generalized SCM [31] (- -). Data for the overlapping solid sphere model (for which no rigorous theories exist) are also shown $(\square)$.

range. The latter observation might have been anticipated given the close association between the definition of the spherical pore model and the assumption of the differential method. At increasing porosities we are simply adding additional spherical pores to a porous matrix. The data for overlapping solid spheres is also shown in the figure, and seen to be quite different from any of the available results. This demonstrates that microstructure (the geometrical nature of the porosity) is an important factor besides the actual value of the porosity.

In Fig. 8, the minimum solid area models and the Ramakrishnan and Arunachalam results [33] are compared with the data. The MSA model for spherical pores performs reasonably well, although underestimating the FEM data for overlapping random spherical pores at low porosities $\phi<0.3$. The MSA model for solid spheres considerably underestimates these data for $\phi<0.3$. The Ramakrishnan and Arunachalam [33] approximation falls between the FEM data for $\phi>0.1$ indicating that it corresponds to neither of the microstructures. For purposes of comparison we also report numerical results obtained using the computational generalized method of cells [ $[$ ]. For a periodic spherical pore the results significantly underestimate the FEM data for overlapping spherical pores at low porosities (and hence the exact dilute result). It is not clear if this is due to the assumptions, or the particular implementation, of the method.

The FEM data for overlapping oblate ellipsoidal pores is compared with the available theories in Fig. 9. The SCM results of $\mathrm{Wu}$ [11] and Berryman [12] underestimate the porosity as a result of underestimating the physical percolation threshold. The Berryman result performs significantly better than does the $\mathrm{Wu}$ result. As for the case of spheres, the differential method matches the data quite closely because of the similarity between the assumptions of the theory and the definition of the model.

We have also compared the Poisson's ratio predicted by the various self-consistent and differential methods to the FEM data for overlapping spherical and ellipsoidal pores. The theoretical results converge to different fixed points (e.g. Fig. 3) in qualitative agreement with the data. But only the differential method provides reasonable agreement with the FEM data (with absolute error less than 0.02 for $\phi \leq 0.4$ and $0.1 \leq \nu_{s} \leq 0.4$ ).

\section{Comparison with Experiment}




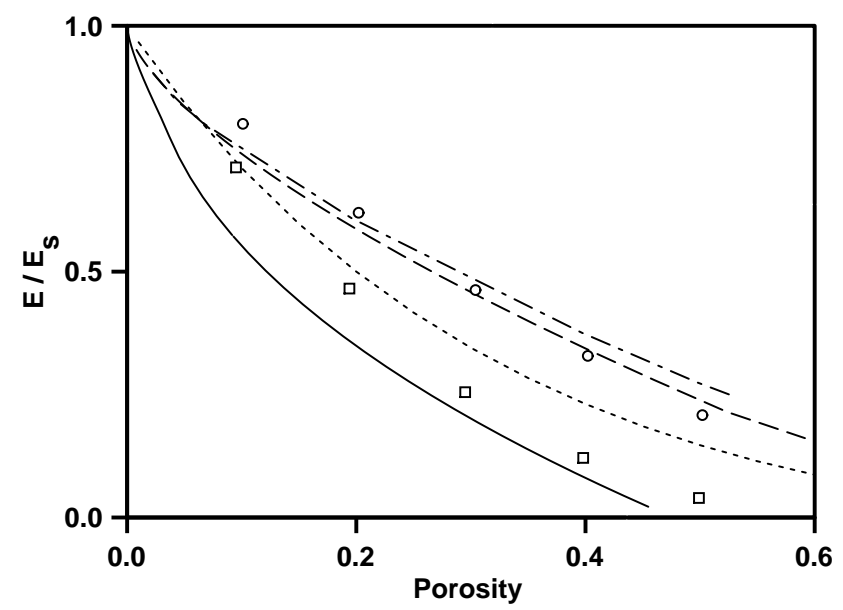

Figure 8: A comparison of the minimum solid area (MSA) models [3] to the FEM data for overlapping spherical pores (o) and solid spheres ( $\square$ ). The MSA solid sphere model ( - ) and MSA porous sphere model (-- ) (in simple cubic packings) are seen to under-estimate the data for low porosities $(\phi<0.3)$. The formula of Ramakrishnan and Arunachalam [33] $E / E_{s}=(1-\phi)^{2} /(1+1.4 \phi)(\cdots)$ and the results of the generalized method of cells for a periodic spherical pore [4] $(-\cdot-)$ are also shown.

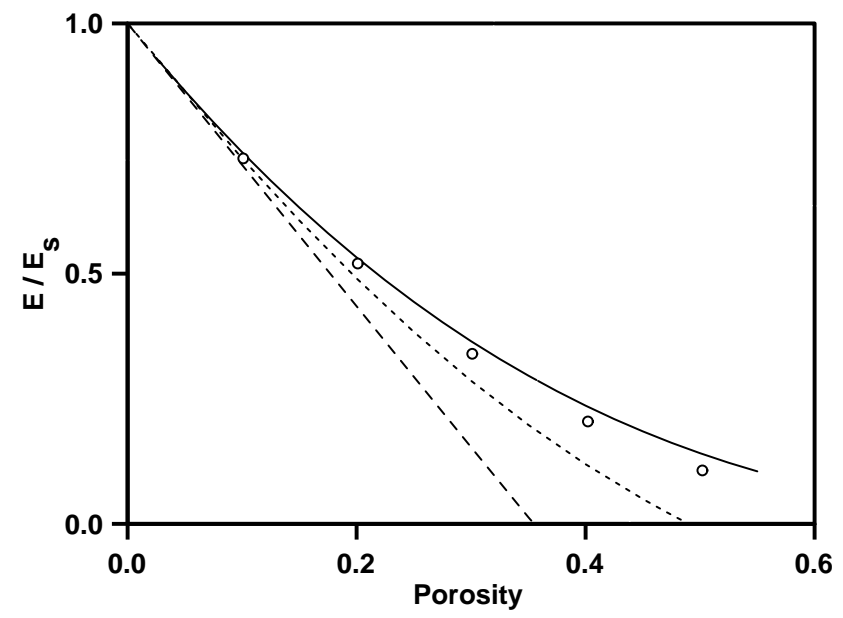

Figure 9: A comparison of different theories to the FEM data for overlapping oblate ellipsoidal pores (०). The lines correspond to the differential method [8] (-) and the self consistent methods of $\mathrm{Wu}[11](---)$ and Berryman [12] $(\cdots)$.

We now use the FEM results to analyze experimental measurements of the elastic properties of porous ceramic materials. The dependence of the elastic moduli on porosity has been the subject of many studies [13, 17, 18]. Data for porous alumina from numerous studies [35] are shown in Fig. 10. The Coble-Kingery [1] material is markedly stiffer than other materials, and is in very good agreement with the FEM results for the overlapping spherical pore model. The pores in the alumina matrix were actually created by the incorporation of a particulate filler [1]], which corresponds well with the definition of the model microstructure. The remaining data closely follow the overlapping solid sphere FEM result for $\phi<0.25$, indicating that the solid alumina phase has the sintered granular morphology exhibited by the model microstructure (Fig.11a). However, Knudsen notes that several of the samples 


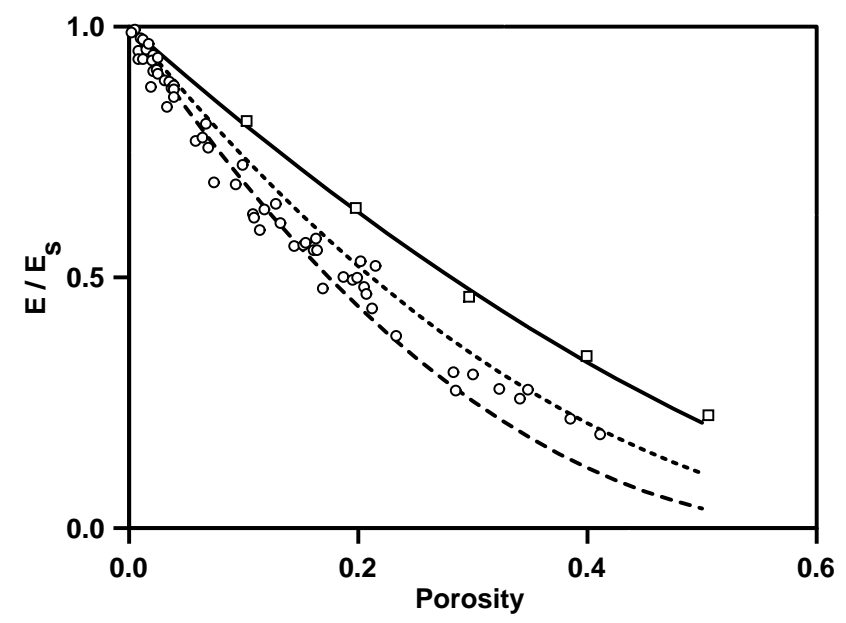

Figure 10: Data for alumina $\left(E_{s}=410 \mathrm{GPa}\right)$ compiled by Knudsen [35] (०). The Coble-Kingery [1]] $\left(E_{s}=386 \mathrm{GPa}\right.$ ) data are also shown $(\square)$. The lines correspond to the FEM theories computed in this paper: overlapping spherical pores $(-)$, overlapping oblate ellipsoidal pores $(\cdots)$ and overlapping solid spheres $(---)$.

summarized were also created using particulate fillers. At higher porosities the solid sphere result underestimates the data. One reason for this might be that the model contains isolated solid spheres which artificially reduce the actual porosity. This was checked and found not to be the case for the porosities studied. Therefore, the solid connections in these samples of porous alumina are likely stiffer than those found in the solid sphere model at porosities $\phi>0.25$. Overlapping spheres can create very sharp "valleys" between a pair of overlapping solid spheres (see Fig. Ia), which would be rounded off in the sintering process, presumably strengthening the solid-solid connection.

Hunter et al. [36, 37, 38, 39] have studied the Young's modulus of several different oxides. In all cases, the porous material was created by sintering a powder of the pure oxide. The results for the Young's modulus are reproduced in Fig. 11. For low porosities $(\phi<0.1)$ all of the data followed the FEM results for overlapping spherical pores. For $\mathrm{Gd}_{2} \mathrm{O}_{3}$ the FEM result continues to provide excellent agreement up to the maximum porosity measured $(\phi=0.4)$ indicating that the microstructure is similar to that of the model (overlapping pores). In contrast, the data for the other three oxides decreases towards the result for overlapping solid spheres indicating a more granular character.

The data of Walsh et al. [25] for porous glass is compared with the FEM results for overlapping spherical pores in Fig. 12. The agreement is good for small to moderate porosities $(\phi<0.3)$, but the FEM results underestimate the data at higher porosities. Walsh et al. point out that the pores in the glass are actually not interconnected (unlike the overlapping pores of the model). This would account for the increased stiffness. It is interesting that the FEM results begin to deviate from the experimental data at the threshold where the pores become macroscopically connected $(\phi=0.3)$. Data for sintered $\mathrm{MgAl}_{2} \mathrm{O}_{2}$ [40] powder is shown in Fig. 13, and is well modeled by the FEM results for overlapping solid spheres. Micrographs of the ceramic indicate a granular structure similar to that of the model microstructure (although the grains appear more like polyhedra, not spheres). 


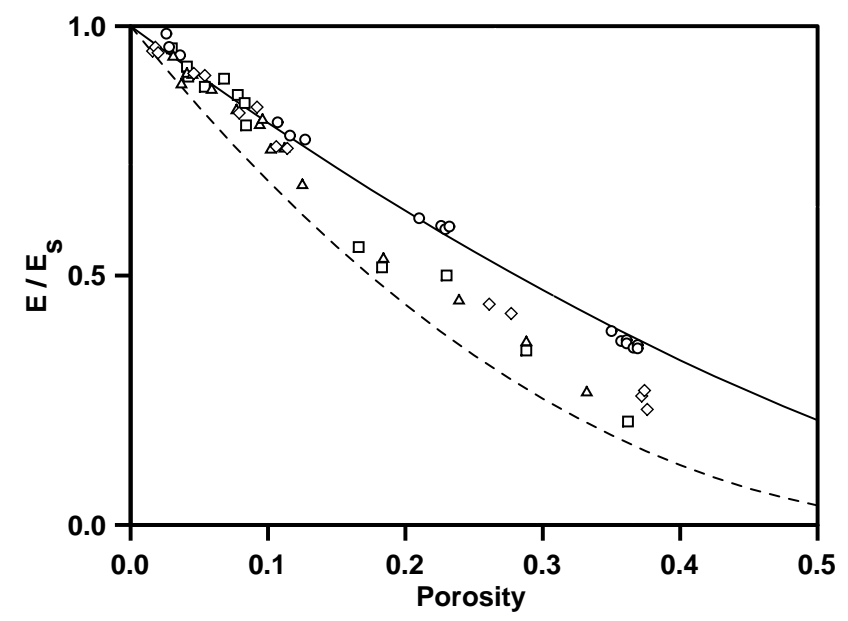

Figure 11: Data for various oxides measured by Hunter et al [36, 37, 38, 39] compared with the FEM theories for overlapping spherical pores (-), and overlapping solid spheres (- - ). $\mathrm{Sm}_{2} \mathrm{O}_{3}$ [36] $E_{s}=145 \mathrm{GPa}(\diamond) ; \mathrm{Lu}_{2} \mathrm{O}_{3}$ [37] $E_{s}=193 \mathrm{GPa}(\Delta) ; \mathrm{Gd}_{2} \mathrm{O}_{3}$ [38] $E_{s}=150 \mathrm{GPa}(\circ) ; \mathrm{HfO}_{2}[39] E_{s}=246 \mathrm{GPa}$ $(\square)$;

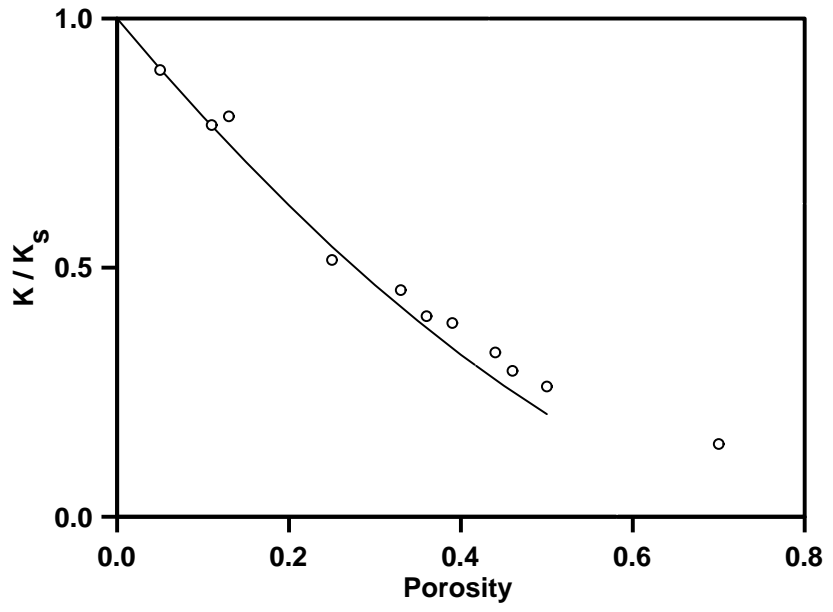

Figure 12: Data for porous glass [25] $\left(K_{s}=46 \mathrm{GPa}, \nu_{s}=0.23\right)$. The line corresponds to the FEM theory for overlapping spherical pores $(\square)$. 


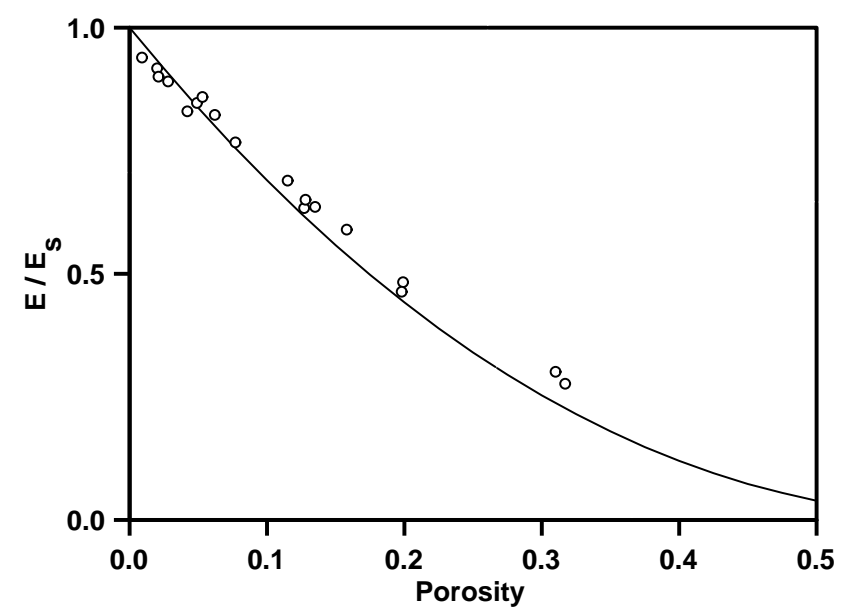

Figure 13: Data for $\mathrm{MgAl}_{2} \mathrm{O}_{4}$ [40]. We used the value $E_{s}=41.2 \times 10^{6} \mathrm{psi}(284 \mathrm{GPa}$ ) indicated on Fig. 3(A) of the reference, rather than the reported value of $E_{s}=43.4 \times 10^{6}$ psi which appears to be a misprint. The line corresponds to the FEM theory for overlapping solid spheres.

\section{Discussion and Conclusions}

We have derived empirical theories for the dependence of the Young's modulus on porosity for three distinct models of porous ceramics, based on careful finite element computations. An advantage of these results over many conventional theories is that they correspond to a priori known microstructures. The dilute result (extended by Coble-Kingery to all porosities), the differential method, and the self consistent methods all have a "built-in" microstructure, but apart from the dilute case, it is not clear what that microstructure is. Therefore, agreement or disagreement with a particular analytic theory neither confirms nor rejects a particular physical model. For the minimum solid area models, the microstructure is exactly known, but the approximation involved in making the Young's modulus directly proportional to the contact area leads to a similar conclusion. Indeed, we found that the MSA models did not provide quantitative agreement with the moduli of the random microstructures studied. We found that the differential method (Eqs. 12 and 13) gave results in reasonable agreement with computed data for the cases of overlapping spherical and ellipsoidal pores, probably due to the similarities between the assumptions of the model and the definitions of the microstructure. Results for the granular model of overlapping solid spheres were not well modeled by any of the analytic theories, demonstrating the importance of finite element techniques in this case of great physical interest.

We have also generated data that shows the dependence of Poisson's ratio on porosity and the solid Poisson's ratio. It is difficult to study this question experimentally because of the inability to vary the Poisson's ratio of the solid independently, and the well known difficulties of accurately measuring the Poisson's ratio at moderate to high porosities [18]. At sufficiently high porosities we find that the Poisson's ratio converges to a fixed non-zero value $\nu_{0}$ irrespective of the solid Poisson's ratio. For overlapping solid spheres $\nu_{0}=0.14$, spherical pores $\nu_{0}=0.22$ and oblate ellipsoidal pores $\nu_{0}=0.16$. This behavior is exact in two dimensions [23, 24] and is exhibited by many of the analytic theories in three dimensions. At present the available experimental data cannot confirm this qualitative behavior [18]. We have shown that the Poisson's ratio does not vanish at high porosities as has been recently argued [19].

It is not simple to attribute our results to features of the solid-pore morphology such as the size, shape, distribution and connectivity of pores or solid grains, since these features have no obvious definition for complex bi-continuous random microstructures. A few general observations can be made, and interpreted in terms of interrelated geometrical and mechanical features of the models. For 
a given porosity, the sintered grain structure of the overlapping solid sphere model is relatively weak. The small solid contacts between spheres and the highly interconnected porosity (which becomes macroscopically connected at $\phi=0.03$ ) lead to a weak structure. We also assume that the valleys which occur between grains will provide sites of large stress concentrations, and consequently, large deformations. In contrast, spherical pores provide high (near optimal) stiffness at a given porosity. The dispersed nature of the porosity (which is macroscopically disconnected for $\phi<0.3$ ) corresponds to a well connected solid matrix. Ellipsoidal pores tend to weaken a structure more than spherical pores due to a combination of a less well connected solid phase (the pores become macroscopically connected at $\phi=0.2$ ), and greater stresses and deformations near the high curvature regions of the ellipsoid.

We have compared our FEM results with several sets of previously published experimental data. In cases where the microstructure of the porous ceramics roughly matched that of the models, the agreement was very good. Since the FEM results correspond to a known microstructure, it was possible to explain deviations in terms of specific microstructural features. Thus, comparison of experimental data with the three computational results provides a useful interpretive tool. Note that a given elastic modulus does not correspond to a particular microstructure. Therefore, it is important to corroborate microstructural interpretations obtained from the elastic moduli with information about the particular material (such as a micrograph). In the future it would be useful to extend this work to higher porosities and to other relevant models (such as non-overlapping porous spheres). It is also possible to use statistical microstructural information obtained from two-dimensional micrographs to generate models [29] that actually mimic physical microstructures.

Acknowledgments A.R. thanks the Fulbright Foundation and Australian Research Council for financial support. We also thank the Partnership for High-Performance Concrete Technology program of the National Institute of Standards and Technology for partial support of this work.

\section{References}

[1] R. L. Coble and W. D. Kingery, "Effect of Porosity on Physical Properties of Alumina", J. Am. Ceram. Soc., 39(11), 377-385 (1956).

[2] E. A. Dean and J. A. Lopez, "Empirical Dependence of Elastic Moduli on Porosity for Ceramic Materials", J. Am. Ceram. Soc., 60(7-8), 345-349 (1977).

[3] R. W. Rice, "Evaluation and extension of physical property-porosity models based on minimum solid area", J. Mater. Sci., 31, 102-118 (1996).

[4] C. T. Herakovich and S. C. Baxter, "Influence of pore geometry on the effective response of porous media”, J. Mater. Sci., 31, 1595-1609 (1999).

[5] Z. Hashin, "Analysis of composite-materials - a survey", J. Appl. Mech., 50, 481-505 (1983).

[6] J. Aboudi, Mechanics of composite materials: a unified micromechanical approach, Elsevier, Amsterdam, 1991.

[7] R. M. Christensen, Mechanics of composite materials, Wiley, New York, 1979.

[8] R. McLaughlin, "A study of the differential scheme for composite materials", Int. J. Eng. Sci., 15, 237-244 (1977).

[9] R. Hill, "A self-consistent mechanics of composite materials", J. Mech. Phys. Solids, 13, 213222 (1965).

[10] B. Budiansky, "On the elastic moduli of some heterogeneous materials", J. Mech. Phys. Solids, 13, 223-227 (1965). 
[11] T. T. Wu, "The effect of inclusion shape on the elastic moduli of a two-phase material", Int. J. Solids Structures, 2, 1-8 (1966).

[12] J. G. Berryman, "Long-wavelength propagation in composite elastic media II. Ellipsoidal inclusions", J. Acoust. Soc. Am., 68(6), 1820-1831 (1980).

[13] R. W. Rice, "Comparison of physical property-porosity behaviour with minimum solid area models”, J. Mater. Sci., 31, 1509-1528 (1996).

[14] S. Torquato, "Random heterogeneous media: Microstructure and improved bounds on effective properties", Appl. Mech. Rev., 44, 37-76 (1991).

[15] E. J. Garboczi and A. R. Day, "An algorithm for computing the effective linear elastic properties of heterogeneous materials: Three-dimensional results for composites with equal phase Poisson ratios", J. Mech. Phys. Solids, 43, 1349-1362 (1995).

[16] J. Poutet, D. Manzoni, F. Hage-chehade, C. G. Jacquin, M. J. Bouteca, J. F. Thovert, and P. M. Adler, "The effective mechanical properties of random porous media", J. Mech. Phys. Solids, 44, 1587-1620 (1996).

[17] N. Ramakrishnan and V. S. Arunachalam, "Effective Elastic Moduli of Ceramic Materials", $J$. Am. Ceram. Soc., 76(11), 2745-52 (1993).

[18] A. R. Boccaccini, "Comment on "Effective Elastic Moduli of Ceramic Materials"”, J. Am. Ceram. Soc., 76(10), 2745-52 (1994).

[19] R. W. Rice, "Comment on "Effective Elastic Moduli of Porous Ceramic Materials"”, J. Am. Ceram. Soc., 78(6), 1711 (1995).

[20] E. J. Garboczi (1998), NIST Internal Report 6269, available at http://ciks.cbt.nist.gov/garboczi, Chapter 2.

[21] H. L. Weissberg, "Effective diffusion coefficient in porous media", J. Appl. Phys., 34, 2636-2639 (1963).

[22] A. P. Roberts and M. Teubner, "Transport properties of heterogeneous materials derived from Gaussian random fields: Bounds and simulation.”, Phys. Rev. E, 51, 4141-4154 (1995).

[23] A. R. Day, K. A. Snyder, E. J. Garboczi, and M. F. Thorpe, "The elastic moduli of sheet containing spherical holes”, J. Mech. Phys. Solids, 40, 1031-1051 (1992).

[24] A. V. Cherkaev, K. A. Lurie, and G. W. Milton, "Invariant properties of the stress in plane elasticity and equivalence classes of composites", Proc. R. Soc. Lond. A, 438, 519-529 (1992).

[25] J. B. Walsh, W. F. Brace, and A. W. England, "Effect of Porosity on Compressibility of Glass", J. Am. Ceram. Soc., 48(12), 605-608 (1965).

[26] E. Garboczi, K. Snyder, J. Douglas, and M. Thorpe, "Geometrical percolation threshold of overlapping ellipsoids”, Phys. Rev. E, 52, 819-828 (1995).

[27] S. Torquato, "Effective stiffness tensor of composite media-I. Exact series expansions", J. Mech. Phys. Solids, 45, 1421-1448 (1997).

[28] S. Torquato, "Effective stiffness tensor of composite media-II. Applications to isotropic dispersions", J. Mech. Phys. Solids, 46, 1411-1440 (1998). 
[29] A. P. Roberts and E. J. Garboczi, "Elastic properties of a tungsten-silver composite by reconstruction and computation", J. Mech. Phys. Solids, 47(10), 2029-2055 (1999).

[30] J. F. MacKenzie, "Elastic constants of a solid containing spherical holes", Proc. Phys. Soc. (London), 63B(1), 2-11 (1960).

[31] R. M. Christensen and K. H. Lo, "Solutions for effective shear properties in three phase sphere and cylinder models", J. Mech. Phys. Solids, 27, 315-330 (1979).

[32] Z. Hashin, “The elastic moduli of heterogeneous materials”, ASME J. Appl. Mech., 29, 143-150 (1962).

[33] N. Ramakrishnan and V. S. Arunachalam, "Effective elastic moduli of porous solids", J. Mater. Sci., 25, 3930-3937 (1990).

[34] F. P. Knudsen, "Dependence of mechanical strength of brittle polycrystalline specimens on porosity and grain size”, J. Am. Ceram. Soc., 42(8), 376-387 (1959).

[35] F. P. Knudsen, "Effect of Porosity on Young's Modulus of Alumina", J. Am. Ceram. Soc., 45(2), 94-95 (1962).

[36] O. Hunter, H. J. Korklan, and R. R. Suchomel, "Elastic Properties of Polycrystalline Monoclinic $\mathrm{Sm}_{2} \mathrm{O}_{3}$ ”, J. Am. Ceram. Soc., 57(6), 267-268 (1974).

[37] O. Hunter and G. E. Graddy, "Porosity Dependence of Elastic Properties of Polycrystalline $\mathrm{Lu}_{2} \mathrm{O}_{3}$ ”, J. Am. Ceram. Soc., 59(1-2), 82 (1976).

[38] J. A. Haglund and O. Hunter, "Elastic Properties of Polycrystalline Monoclinic $\mathrm{Gd}_{2} \mathrm{O}_{3}$ ", J. Am. Ceram. Soc., 56(6), 327-330 (1973).

[39] S. L. Dole, O. Hunter, and F. W. Calderwood, "Elastic Properties of Stabilized $\mathrm{HfO}_{2}$ Compositions", J. Am. Ceram. Soc., 63(3-4), 136-139 (1980).

[40] D. F. Porter, J. S. Reed, and D. Lewis, "Elastic Moduli of Refractory Spinels", J. Am. Ceram. Soc., 60(7-8), 345-349 (1977). 